2PA16

\title{
Control of Plasma Parameters in RF Negative Ion Sources $^{\dagger}$
}

\author{
Y. Tauchi, O. Fukumasa
}

Yamaguchi University, Tokiwadai 2-16-1, Ube 755-8611, Japan

A long lifetime neutral beam injector (NBI) system is necessary for heating future fusion plasmas. Therefore negative ion sources for NBI system should be constructed by using high frequency discharge plasmas. Radio frequency (RF, 13.56MHz.) discharge plasma is one of the most promising candidate for long lifetime ion sources.

On the other hand, in hydrogen plasmas, most $\mathrm{H}^{-}$ions are produced by the following two-step process:

1) $\mathrm{H}_{2}+\mathrm{ef}(\mathrm{E}>2 \mathrm{OeV}) \rightarrow \mathrm{g}_{2}\left(\mathrm{v}^{\prime \prime}\right)+e f$,

2) $\mathrm{H}_{2}\left(\mathrm{v}^{\prime \prime}\right)+\mathrm{es}(\mathrm{Te} \cong 1 \mathrm{eV}) \rightarrow \mathrm{g}^{-}+\mathrm{H}$.

$\mathrm{H}^{-}$ions are also destroyed easily by collisions with high energy electrons. Therefore, electron energy in source plasmas must be controlled well for optimizing $\mathrm{H}^{-}$production, and DC discharge plasmas are well optimized by using the magnetic filter(MF)[1].

So far, we studied production and control of ECR and RF (i.e. capacitively coupled plasma: CCP) plasmas by using MF method. But plasma conditions are not well optimized for $\mathrm{H}^{-}$production[2], especially in controlling electron temperature, $T e$, of $C C P[3]$. In this paper, we further study control of RF plasmas with the MF or mesh grid(MG) bias method. Te control with the MG bias method [4] is well used in plasma processing.

RF negative ion source chamber (210 $\mathrm{mm}$ in diameter $450 \mathrm{~mm}$ in length) made of stainless steel is a conventional line-cusp volume source. This chamber is divided into two regions by the MF or the MG. i.e. plasma source region with disk antenna and diffused plasma region. RF power, $W_{R F}$, applied at disk antenna is varied from $10 \mathrm{~W}$ to $200 \mathrm{~W}$. Hydrogen pressure, $P$, is varied from $3 \mathrm{~m}$ Torr to $10 \mathrm{~m}$ Torr.

When grid potential, $V_{G}$, is set at a certain bias voltage under $-20 \mathrm{~V}, \mathrm{Te}$ is decreased below about $\mathrm{leV}$ at diffused plasma region. For example, when $W_{R F}=200 \mathrm{~W}, V_{G}=-25 \mathrm{~V}$ and $P=5 \mathrm{mTorr}$, plasma parameters of source region are $N e=9 \times 10^{8} \mathrm{~cm}^{-3}$ and $T e$ $=5 \mathrm{eV}$. On the other hand, plasma parameters of diffused region are $N e=4 \times 10^{8} \mathrm{~cm}^{-3}$ and $T \mathrm{e}=1 \mathrm{eV}$. Thus, we have confirmed that, for optimizing $\mathrm{H}^{-}$production $\mathrm{RF}$ plasmas are more controlled by using MG bias method compared with MF method. Now, we study the relationship between extraction of $\mathrm{H}^{-}$currents and plasma parameters by using MG bias method. Details are reported in the conference.

[1] O. Fukumasa et al.: Rev. Sci. Instrum. 63(1992)2696.

2] O. Fukumasa and M. Matsumori: Rev. Sci. Instrum. 71(2000) 935.

[3] T. Tasaka, Y. Tauchi O. Fukumasa and Y. Takeiri: PST-0185(in Japanese)

[4] K. Kato, S. Izuka and N. Sato: Appl. Phys. Lett. 65(1994)816

\footnotetext{
$\dagger_{\text {tauchi@ee.yamaguchi-u.ac.jp }}$
} 